\title{
State Estimators for Uncertain Linear Systems with Different Disturbance/Noise Using Quadratic Boundedness
}

\author{
Longge Zhang, ${ }^{1,2}$ Xiangjie Liu, ${ }^{2}$ and Xiaobing Kong ${ }^{2}$ \\ ${ }^{1}$ Department of Mathematics and Physics, North China Electric Power University, Baoding 071003, China \\ 2 State Key Laboratory of Alternate Electrical Power System with Renewable Energy Sources, North China \\ Electric Power University, Beijing 102206, China
}

Correspondence should be addressed to Longge Zhang, longgexd@163.com

Received 28 February 2012; Revised 8 April 2012; Accepted 10 April 2012

Academic Editor: Baocang Ding

Copyright (C) 2012 Longge Zhang et al. This is an open access article distributed under the Creative Commons Attribution License, which permits unrestricted use, distribution, and reproduction in any medium, provided the original work is properly cited.

This paper designs state estimators for uncertain linear systems with polytopic description, different state disturbance, and measurement noise. Necessary and sufficient stability conditions are derived followed with the upper bounding sequences on the estimation error. All the conditions can be expressed in the form of linear matrix inequalities. A numerical example is given to illustrate the effectiveness of the approach.

\section{Introduction}

In many control systems, the state variables are usually not accessible for direct connection. In this case, it is necessary to design a state estimator, so that its output will generate an estimate of those states. Generally speaking, there are two kinds of estimators for dynamic systems: observers and filters. The former is under the supposition of the perfect knowledge of system and measurement equations, and the latter can be applied to the system with disturbance. Many literatures focus on the design of state estimators for linear system, for example, a sliding mode and a disturbance detector for a discrete Kalman filter [1], the quantized measurement method [2], the least squares estimation for linear singular systems [3], stochastic disturbances and deterministic unknown inputs on linear time-invariant systems [4], and the bounded disturbances on a dynamic system [5].

The concept of quadratic boundedness (QB) is first defined for an uncertain nonlinear dynamical system [6], and then its necessary and sufficient conditions for a class of nominally linear systems [7] and a class of linear systems which contain norm-bounded uncertainties 
[8] are obtained. For discrete system, QB is applied mainly two regions: the receding horizon control (RHC) and the design of estimator. In RHC research, Ding utilizes QB to characterize the stability properties of the controlled system [9-13]. Alessandri et al. find the upper bounds on the norm of the estimation error by means of invariant sets, and these upper bounds can be expressed in terms of linear matrix inequalities [14]. Paper in [5] designs a filter by searching a suitable tradeoff between the transient and asymptotic behaviors of the estimation error. The designed filter is for the linear discrete systems with the identical state disturbance and measurement noise. For the discrete linear systems, the disturbance and noise are different in general. Nevertheless, little work has been done on the design of the state estimators for uncertain linear systems with different disturbance/noise. So how to design state estimators for uncertain linear systems with different state disturbance and measurement noise is important work.

The existing research work on state estimation usually constructs a filter for uncertain systems with bounded disturbance/noise, with no consideration of input or state constraint. Since the disturbance and noise are not assumed exactly identical, the stability station of the estimator is different from that in the paper [5]. For the above reasons, the situation becomes more complicated and the extension of the method is not straightforward.

This paper designs state estimators for uncertain linear systems with polytopic description. The problem is constructed in the form of linear matrix inequalities (LMIs). The organization of the paper is as follows. The earlier results are presented in the Section 2. The new robust estimator for uncertain linear systems with different disturbance/noise is designed in Section 3. A numerical simulation example is followed in Section 4. And some conclusions are given in the end.

Notations. For any vector $x$ and a positive-defined matrix $Q, \varepsilon_{Q}$ is the ellipsoid which is defined as $\left\{x \mid x^{\prime} Q x \leq 1\right\} ; Q^{\prime}$ is the transpose of matrix $Q$. $\|x\|$ is the Euclid norm of vector $x$. The symbol ${ }^{*}$ induces a symmetric structure in LMIs.

\section{Earlier Results}

In this section, some results presented by Alessandri et al. $[5,14]$ are briefly introduced.

For a given discrete-time dynamic system,

$$
x_{t+1}=A_{t} x_{t}+G_{t} w_{t}, \quad t=0,1, \ldots,
$$

where $x_{t} \in R^{n}$ is the state vector and $w_{t} \in \mathcal{\varepsilon}_{Q} \subset R^{p}$ is the noise vector. The definition of strictly quadratically bounded with a common Lyapunov matrix of a system and positively invariant set are defined by Alessandri et al. [5, 14], and the following theorem is proved.

Theorem 2.1 (see [14]). The following facts are equivalent:

(i) System (2.1) is strictly quadratically bounded with a common Lyapunov matrix $P>0$ for all allowable $w_{t} \in \mathcal{E}_{Q}$ and $\left(A_{t}, G_{t}\right) \in \varphi, t=0,1, \ldots$, where $\varphi$ is a known bounded set.

(ii) The ellipsoid $\mathcal{\varepsilon}_{P}$ is a positively invariant set for system (2.1) for all allowable $w_{t} \in \mathcal{\varepsilon}_{Q}$ and $\left(A_{t}, G_{t}\right) \in \varphi, t=0,1, \ldots$

(iii) There exists $\alpha_{t} \in(0,1)$ such that for any $\left(A_{t}, G_{t}\right) \in \varphi$, 
Journal of Applied Mathematics

$$
\left[\begin{array}{cc}
A_{t}^{\prime} P A_{t}-P+\alpha_{t} P & A_{t}^{\prime} P G_{t} \\
* & G_{t}^{\prime} P G_{t}-\alpha_{t} Q
\end{array}\right] \leq 0 .
$$

For a discrete-time linear system with the same state disturbance and noise,

$$
\begin{gathered}
x_{t+1}=A_{t} x_{t}+B_{t} w_{t}, \\
y_{t}=C_{t} x_{t}+D_{t} w_{t}, \\
z_{t}=L_{t} x_{t},
\end{gathered}
$$

for $t=0,1, \ldots$, where $x_{t} \in R^{n}, y_{t} \in R^{m}, z_{t} \in R^{r}$ are the state vector, the measured output and the signal will be estimated, respectively, and $w_{t} \in \varepsilon_{Q} \subset R^{p}$ is the disturbance/noise vector. $A_{t}, B_{t}, C_{t}, D_{t}, L_{t}$ are the system matrixes with the proper dimensions. We consider the disturbance/noise to be unknown, and the system matrixes are supposed to be unknown and time varying but belonging to a polytopic set $D$, that is, $\left(A_{t}, B_{t}, C_{t}, D_{t}, L_{t}\right) \in D, t=0,1, \ldots$, where

$$
p \triangleq\left\{(A, B, C, D, L)=\sum_{i=1}^{N} \lambda_{i}\left(A^{(i)}, B^{(i)}, C^{(i)}, D^{(i)}, L^{(i)}\right) ; \sum_{i=1}^{N} \lambda_{i}=1, \lambda_{i} \geq 0, i=1,2, \ldots N\right\}
$$

Here $\left(A^{(i)}, B^{(i)}, C^{(i)}, D^{(i)}, L^{(i)}\right) i=1,2, \ldots N$ are vertexes of the polytope $D$.

Definition 2.2 (see [5]). A sequence of vectors $\xi_{t}$ is said to be exponentially bounded with constants $\beta \in(0,1), k_{1} \geq 0$, and $k_{2}>-k_{1}$ if

$$
\left\|\xi_{t}\right\| \leq k_{1}+k_{2}(1-\beta)^{t}, \quad t=0,1, \ldots
$$

It is easy to see that $\beta$ determines the convergence speed and $k_{1}^{1 / 2}$ represents an upper bound of the sequence $\xi_{t}$.

Theorem 2.3 (see [5]). Consider two scalars $\alpha \in(0,1)$ and $\gamma>0$. The following facts are equivalent.

(i) There exist $\widehat{A}, \widehat{B}, \widehat{L}$, and $P>0$ such that the following conditions are satisfied for any $(A, B, C, D, L) \in D$ :

$$
\begin{gathered}
\tilde{C} P^{-1} \widetilde{C}^{\prime}-\gamma^{2} I<0 \\
{\left[\begin{array}{cc}
A^{\prime} P A-P+\alpha P & A^{\prime} P G \\
* & G^{\prime} P G-\alpha Q
\end{array}\right]<0 .}
\end{gathered}
$$


(ii) There exist $V, W, X>0, Y>0$, and $Z$ such that

$$
\begin{gathered}
{\left[\begin{array}{cccc}
\gamma^{2} I & L-W & L \\
* & X & X \\
* & * & Y
\end{array}\right]>0,} \\
{\left[\begin{array}{ccccc}
(1-\alpha) X & (1-\alpha) X & 0 & A^{\prime} X & A^{\prime} Y+C^{\prime} Z^{\prime}+V^{\prime} \\
* & (1-\alpha) Y & 0 & A^{\prime} X & A^{\prime} Y+C^{\prime} Z^{\prime} \\
* & * & \alpha Q & B^{\prime} X & D^{\prime} Z+B^{\prime} Y \\
* & * & * & X & X \\
* & * & * & * & Y
\end{array}\right]>0,}
\end{gathered}
$$

for $(A, B, C, D, L)=\left(A^{(i)}, B^{(i)}, C^{(i)}, D^{(i)}, L^{(i)}\right), i=1,2, \ldots N$.

Then, the cost $J(r, \alpha) \triangleq \mu \gamma-(1-\mu) \alpha$ can be minimized over $V, W, X>0, Y>0, Z, \alpha \in(0,1)$ and $r>0$ under the constrains (2.7).

\section{A Robust Estimator for Uncertain Linear Systems with Different Noises}

Let us consider the discrete-time linear system with different disturbance/noise:

$$
\begin{gathered}
x_{t+1}=A_{t} x_{t}+B_{t} w_{t}, \\
y_{t}=C_{t} x_{t}+D_{t} v_{t}, \\
z_{t}=L_{t} x_{t},
\end{gathered}
$$

where the matrixes and vectors are the same as (2.3) except $w_{t} \in \varepsilon_{Q_{1}}, v_{t} \in \varepsilon_{Q_{2}}$, which are the vectors of the state disturbance and measurement noise, respectively.

To estimate the signal $z_{t}$, the linear filter is introduced which has the following form:

$$
\begin{gathered}
\widehat{x}_{t+1}=\widehat{A} \widehat{x}_{t}+\widehat{B} y_{t}, \\
\widehat{z}_{t}=\widehat{L} \widehat{x}_{t},
\end{gathered}
$$

for $t=0,1, \ldots$, where $\widehat{x}_{t} \in R^{n}$ is the filter state vector and $\widehat{z}_{t} \in R^{r}$ is the estimation of the signal $z_{t}$.

Define the estimation error $e_{t}$, the augmented state vector, and the augmented disturbance/noise as

$$
e_{t} \triangleq z_{t}-\widehat{z}_{t}, \quad \tilde{x}_{t} \triangleq\left[\begin{array}{l}
x_{t} \\
\widehat{x}_{t}
\end{array}\right], \quad \tilde{w}_{t}=\left[\begin{array}{c}
w_{t} \\
v_{t}
\end{array}\right]
$$


and the dynamic system associated with the estimation error

$$
\begin{gathered}
\tilde{x}_{t+1}=\frac{\left[\begin{array}{cc}
A & 0 \\
\widehat{B} C_{t} & \widehat{A}
\end{array}\right]}{\tilde{A}} \tilde{x}_{t}+\frac{\left[\begin{array}{cc}
B_{t} & 0 \\
0 & \widehat{B} D_{t}
\end{array}\right]}{\tilde{B}} \\
e_{t}=\frac{\left[L_{t}-\widehat{L}\right] \tilde{x}_{t},}{\tilde{C}},
\end{gathered}
$$

for $t=0,1, \ldots$.

The objective is to find an estimate $\widehat{z}_{t}$ of the signal $z_{t}$ such that the estimation error $e_{t}=$ $z_{t}-\widehat{z}_{t}$ is exponentially bounded for any $x_{0} \in R^{n}, w_{t} \in \mathcal{E}_{Q_{1}}, v_{t} \in \mathcal{E}_{Q_{2}}$, and $\left(A_{t}, B_{t}, C_{t}, D_{t}, L_{t}\right) \in$ $p, t=0,1, \ldots$. Then the following problem has to be solved.

Problem 1. Find matrices $\widehat{A}, \widehat{B}, \widehat{L}$ such that, for any $x_{0} \in R^{n}, w_{t} \in \mathcal{E}_{Q_{1}}, v_{t} \in \mathcal{E}_{Q_{2}}$ and $\left(A_{t}, B_{t}, C_{t}, D_{t}, L_{t}\right) \in D, t=0,1, \ldots ;$ the estimation error $e_{t}$ is exponentially bounded with constants $\beta \in(0,1), k_{1} \geq 0$, and $k_{2}>-k_{1}$.

In order to solve Problem 1, we now exploit the results on quadratic boundedness. More specifically, the following proposition holds.

Proposition 3.1. Suppose there exist matrices $\widehat{A}, \widehat{B}, \widehat{L}$, a symmetric matrix $P>0$, and two scalars $\gamma>0$ and $\alpha \in(0,1)$ such that, for any $(A, B, C, D, L) \in D$,

$$
\begin{gathered}
\tilde{C} P^{-1} \tilde{C}^{\prime}-\gamma^{2} I<0, \\
{\left[\begin{array}{cc}
\tilde{A} P \tilde{A}-P+\alpha P & \widetilde{A}^{\prime} P \widetilde{B} \\
* & \widetilde{B}^{\prime} P \widetilde{B}-\alpha R
\end{array}\right]<0,}
\end{gathered}
$$

where $R=\operatorname{diag}\left\{Q_{1}, Q_{2}\right\}$. Then, for any $x_{0} \in R^{n}, w_{t} \in \mathcal{E}_{Q_{1}}, v_{t} \in \mathcal{\varepsilon}_{Q_{2}}$, and $\left(A_{t}, B_{t}, C_{t}, D_{t}, L_{t}\right) \in$ $D, t=0,1, \ldots$, the estimation error is exponentially bounded with constants

$$
\beta=\alpha, \quad k_{1}=\gamma^{2}, \quad k_{2}=\gamma^{2}\left(\tilde{x}_{0}^{\prime} P \tilde{x}_{0}-1\right) .
$$

Hence the matrices $\widehat{A}, \widehat{B}$, and $\widehat{L}$ are a solution of Problem 1 .

Remark 3.2. We can see that condition (3.7) ensures system (3.1) is strictly bounded with a common Lyapunov matrix $P$, and from Corollary 2 of paper [5], it is clearly, $\gamma$ is a bound. But its feasibility cannot be easily verified. The following theorem transposes them into the equivalent LMI conditions.

Theorem 3.3. Consider two scalars $\alpha \in(0,1)$ and $\gamma>0$. The following facts are equivalent.

(i) There exist $\widehat{A}, \widehat{B}, \widehat{L}$, and $P>0$ such that conditions (3.6) and (3.7) are satisfied for any $(A, B, C, D, L) \in D$.

(ii) There exist $V, W, X>0, Y>0$, and $Z$ such that 


$$
\begin{gathered}
{\left[\begin{array}{cccc}
r^{2} I & L-W & L \\
* & X & X \\
* & * & Y
\end{array}\right]>0,} \\
{\left[\begin{array}{cccccc}
(1-\alpha) X & (1-\alpha) X & 0 & 0 & A^{\prime} X & A^{\prime} Y+C^{\prime} Z^{\prime}+V^{\prime} \\
* & (1-\alpha) Y & 0 & 0 & A^{\prime} X & A^{\prime} Y+C^{\prime} Z^{\prime} \\
* & * & \alpha Q_{1} & 0 & B^{\prime} X & B^{\prime} Y \\
* & * & 0 & \alpha Q_{2} & 0 & D^{\prime} Z \\
* & * & * & * & X & X \\
* & * & * & * & * & Y
\end{array}\right]>0 .}
\end{gathered}
$$

Proof. The proof of (3.6) $\Leftrightarrow(3.9)$ is the same as Theorem 2 of reference [5]; here it is omitted for brevity.

$(3.7) \Rightarrow(3.10)$ suppose the condition (3.7) is satisfied. The matrix $P$ and matrix $P^{-1}$ are partitioned as

$$
P=\left[\begin{array}{ll}
P_{11} & P_{12} \\
P_{21} & P_{22}
\end{array}\right], \quad P^{-1}=\left[\begin{array}{ll}
S_{11} & S \\
S_{21} & S
\end{array}\right],
$$

with $P_{11} \in R^{n \times n}$ and $S_{11} \in R^{n \times n}$. Clearly $P P^{-1}=P^{-1} P=I$, so we have

$$
S_{12} P_{12}^{\prime}=I-S_{11} P_{11}, \quad S_{11} P_{12}+S_{12} P_{22}=0 .
$$

Moreover, (3.7) is strict inequality, and we can assume, without loss of generality, that $I-$ $S_{11} P_{11}$ is invertible [15]. Hence $S_{12}$ and $P_{12}$ are invertible. Using the Schur complement, we can rewrite (3.7) as

$$
\left[\begin{array}{ccc}
(1-\alpha) P & 0 & \tilde{A}^{\prime} P \\
0 & \alpha R & \tilde{B}^{\prime} P \\
* & * & P
\end{array}\right]>0
$$

\section{Define}

$$
T \triangleq\left[\begin{array}{ccc}
H^{\prime} & 0 & 0 \\
0 & I & 0 \\
0 & 0 & H^{\prime}
\end{array}\right],
$$


where $H^{\prime}=\left[\begin{array}{cc}I & I \\ S_{12}^{\prime} S_{11}^{-1} & 0\end{array}\right]$, so

$$
\begin{aligned}
& T^{\prime}\left[\begin{array}{ccc}
(1-\alpha) P & 0 & \tilde{A}^{\prime} P \\
0 & \alpha R & \widetilde{B}^{\prime} P \\
* & * & P
\end{array}\right] T=\left[\begin{array}{ccc}
H & 0 & 0 \\
0 & I & 0 \\
0 & 0 & H
\end{array}\right]\left[\begin{array}{ccc}
(1-\alpha) P & 0 & \tilde{A}^{\prime} P \\
0 & \alpha R & \widetilde{B}^{\prime} P \\
* & * & P
\end{array}\right]\left[\begin{array}{ccc}
H^{\prime} & 0 & 0 \\
0 & I & 0 \\
0 & 0 & H^{\prime}
\end{array}\right] \\
&= {\left[\begin{array}{ccc}
(1-\alpha) H P & 0 & H \tilde{A}^{\prime} P \\
0 & \alpha R & \widetilde{B}^{\prime} P \\
H P A & H P \widetilde{B} & H P
\end{array}\right]\left[\begin{array}{ccc}
H^{\prime} & 0 & 0 \\
0 & I & 0 \\
0 & 0 & H^{\prime}
\end{array}\right]=\left[\begin{array}{ccc}
(1-\alpha) H P H^{\prime} & 0 & H \tilde{A}^{\prime} P H^{\prime} \\
0 & \alpha R & \widetilde{B}^{\prime} P H^{\prime} \\
H P A H^{\prime} & H P \widetilde{B} & H P H^{\prime}
\end{array}\right], } \\
& H P H^{\prime}=\left[\begin{array}{ccc}
I & S_{11}^{-1} S_{12} \\
0
\end{array}\right]\left[\begin{array}{cc}
P_{11} & P_{12} \\
P_{21} & P_{22}
\end{array}\right]\left[\begin{array}{cc}
I & I \\
S_{12}^{\prime} S_{11}^{-1} & 0
\end{array}\right] \\
&= {\left[\begin{array}{c}
P_{11}+S_{11}^{-1} S_{12} P_{21}+\left(P_{12}+S_{11}^{-1} S_{12} P_{22}\right) S_{12}^{\prime} S_{11}^{-1} \\
P_{11}+P_{12} S_{12}^{\prime} S_{11}^{-1}+S_{11}^{-1} S_{12} P_{21} \\
P_{11}
\end{array}\right] . }
\end{aligned}
$$

Using condition (3.12), we can get

$$
H P H^{\prime}=\left[\begin{array}{ll}
S_{11}^{-1} & S_{11}^{-1} \\
S_{11}^{-1} & P_{11}
\end{array}\right]=\left[\begin{array}{cc}
X & X \\
X & Y
\end{array}\right],
$$

where $X \triangleq S_{11}^{-1}, Y \triangleq P_{11}$ :

$$
\begin{aligned}
H \widetilde{A}^{\prime} P H^{\prime} & =\left[\begin{array}{cc}
I & S_{11}^{-1} S_{12} \\
I & 0
\end{array}\right]\left[\begin{array}{cc}
A^{\prime} & C^{\prime} \widehat{B}^{\prime} \\
0 & \widehat{A}^{\prime}
\end{array}\right]\left[\begin{array}{ll}
P_{11} & P_{12} \\
P_{21} & P_{22}
\end{array}\right]\left[\begin{array}{cc}
I & I \\
S_{12}^{\prime} S_{11}^{-1} & 0
\end{array}\right] \\
& =\left[\begin{array}{cc}
A^{\prime} & C^{\prime} \widehat{B}^{\prime}+S_{11}^{-1} S_{12} \widehat{A}^{\prime} \\
A^{\prime} & C^{\prime} \widehat{B}^{\prime}
\end{array}\right]\left[\begin{array}{ll}
P_{11}+P_{12} S_{12}^{\prime} S_{11}^{-1} & P_{11} \\
P_{21}+P_{22} S_{12}^{\prime} S_{11}^{-1} & P_{21}
\end{array}\right] \\
& =\left[\begin{array}{cc}
A^{\prime} X & A^{\prime} Y+C^{\prime} Z^{\prime}+V^{\prime} \\
A^{\prime} X & A^{\prime} Y+C^{\prime} Z^{\prime}
\end{array}\right] .
\end{aligned}
$$

Here we define $V \triangleq P_{12} \widehat{A} S_{12}^{\prime} S_{11}^{-1}, Z \triangleq P_{12} \widehat{B}$ :

$$
\begin{aligned}
\widetilde{B}^{\prime} P H^{\prime} & =\left[\begin{array}{cc}
B^{\prime} & 0 \\
0 & D^{\prime} \widehat{B}^{\prime}
\end{array}\right]\left[\begin{array}{cc}
P_{11} & P_{12} \\
P_{21} & P_{22}
\end{array}\right]\left[\begin{array}{cc}
I & I \\
S_{12}^{\prime} S_{11}^{-} & 0
\end{array}\right] \\
& =\left[\begin{array}{cc}
B^{\prime} P_{11} & B^{\prime} P_{12} \\
D^{\prime} \widehat{B}^{\prime} P_{21} & D^{\prime} \widehat{B}^{\prime} P_{22}
\end{array}\right]\left[\begin{array}{cc}
I & I \\
S_{12}^{\prime} S_{11}^{-} & 0
\end{array}\right]=\left[\begin{array}{cc}
B^{\prime} P_{11}+B^{\prime} P_{12} S_{12}^{\prime} S_{11}^{-1} & B^{\prime} P_{11} \\
D^{\prime} \widehat{B}^{\prime} P_{21}+D^{\prime} \widehat{B}^{\prime} P_{22} S_{12}^{\prime} S_{11}^{-1} & D^{\prime} \widehat{B}^{\prime} P_{21}
\end{array}\right] \\
& =\left[\begin{array}{cc}
B^{\prime} X & B^{\prime} Y \\
0 & D^{\prime} Z^{\prime}
\end{array}\right] .
\end{aligned}
$$

So we can get condition (3.10). 


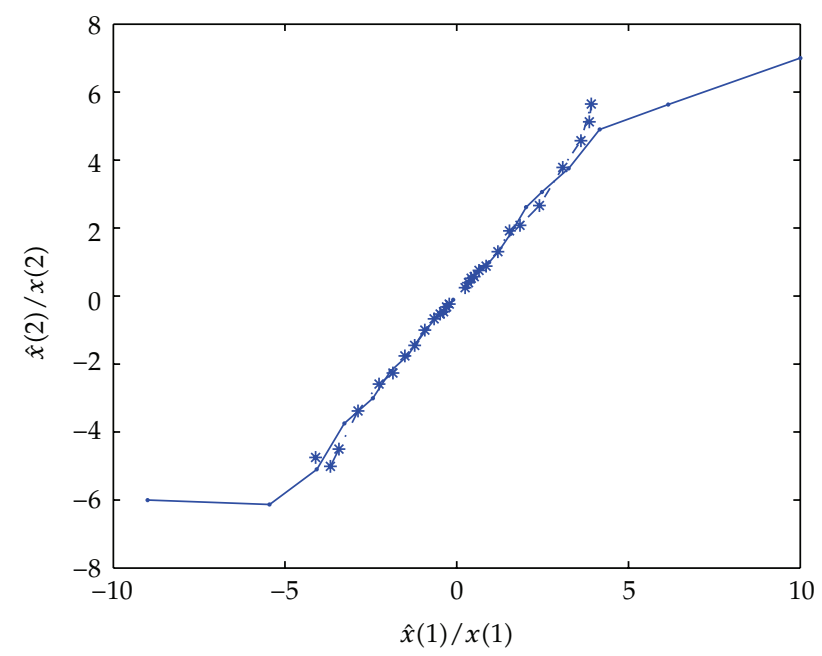

Figure 1: The state and estimator trajectories.

$(3.10) \Rightarrow(3.7)$ suppose there exist $V, X>0, Y>0$ and $Z$ satisfied condition (3.4), as condition (3.4) holds at every vertex $\left(A^{(\mathrm{i})}, B^{(\mathrm{i})}, C^{(\mathrm{i})}, D^{(\mathrm{i})}, L^{(\mathrm{i})}\right)$ of the polytope $D$, and it also holds for every system matrice $(A, B, C, D, L) \in \mathcal{D}$.

We can obtain $\left[\begin{array}{ll}X & X \\ X & Y\end{array}\right]>0$ from condition (3.10), and based on the Schur complement, the result of $I-X^{-1} Y<0$ can be deduced. Then there exist two square invertible matrixes $M$ and $N$ such that $M^{\prime} N^{\prime}=I-X^{-1} Y$. Choosing $P_{11}=Y, S_{11}=X^{-1}, S_{12}^{\prime}=M$ and $P_{12}=N$, condition (3.7) can be obtained by premultiplying and postmultiplying condition (3.10) by $\left(T^{\prime}\right)^{-1}$ and $T^{-1}$. If we apply the change of variable

$$
\begin{gathered}
\widehat{A}=N^{-1} V X^{-1} M^{-1}, \quad \widehat{B}=N^{-1} Z, \\
\widehat{L}=W X^{-1} M^{-1}, \quad P=\left[\begin{array}{cc}
Y & N \\
N^{\prime} & -N^{\prime} X^{-1} M^{-1}
\end{array}\right] .
\end{gathered}
$$

so, we can get the linear filter (3.2).

Remark 3.4. In paper [5], the estimators for uncertain systems propose that the state disturbance and measurement noise are identical with the time. Ordinarily, they are different in practice, so the result in our paper is the general case.

\section{A Numerical Example}

Let us consider the system [12] in the form of (3.1) with

$$
\begin{gathered}
A_{t}=\left[\begin{array}{cc}
0.385 & 0.33 \\
0.21+a_{t} & 0.59
\end{array}\right], \quad B_{t}=\left[\begin{array}{l}
0.3 \\
0.3
\end{array}\right], \\
C_{t}=\left[\begin{array}{ll}
0.2 & 0.2+a_{t}
\end{array}\right], \quad D_{t}=0.3, \quad L_{t}=[10],
\end{gathered}
$$




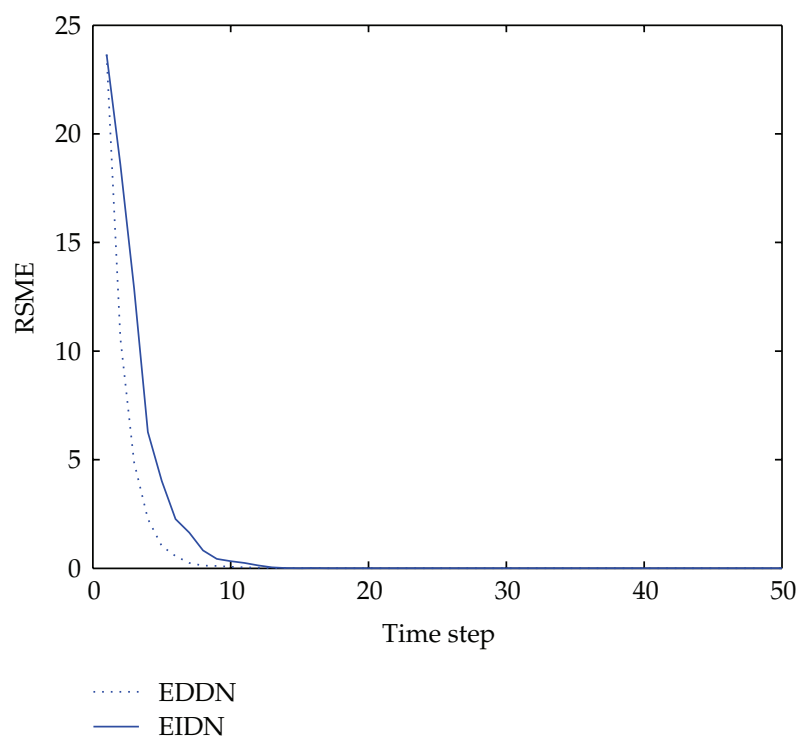

Figure 2: Plots of the RSME for the considered filters.

where $a_{t}$ is an uncertain parameter satisfying $\left|a_{t}\right| \leq 0.11$. Suppose the state disturbance satisfies the condition $\left|w_{t}\right| \leq 0.25$ and the measurement noise satisfies $\left|v_{t}\right| \leq 0.1$ (i.e., $\left.Q_{1}=16, Q_{2}=100\right)$.

As this kind of uncertainty is of the polytopic type described in Section 3, the proposed method is used to obtain a linear filter. In the context, we will refer to this filter as the "filter with different disturbance/noise" (FDDN). Choose two sets of initial states: $\widehat{x}_{0}=$ $\left\{\left[\begin{array}{ll}8 & 6\end{array}\right]^{T},\left[\begin{array}{ll}-11 & 7\end{array}\right]^{T}\right\}, x_{0}=\left\{\left[\begin{array}{ll}10 & 7\end{array}\right]^{T},\left[\begin{array}{ll}-9 & -6\end{array}\right]^{T}\right\}$. The resulting state trajectories are shown in Figure 1 by the marked solid line, followed with the estimator state trajectories shown by the marked dotted line. Figure 1 indicates that the designed estimator can track the systems' states effectively.

The performance of the filter can be further studied by using an average measure of the estimation error, such as the expected quadratic estimation error. Comparison was then made between the FDDN and the "filter with identity disturbance/noise" (FIDN), to evaluate the performance achieved when the different disturbance/noise is taken into account in the synthesis of the filter. At each time instant, the uncertain parameters were chosen to be within, with equal probability, one of their limit values. We assumed $x_{0}, w_{t}$ and $v_{t}, t=0,1, \ldots$, to be independent random vectors, and the initial states are in the ball of radius 10 (i.e., $\left.\left\|x_{0}\right\| \leq 10\right)$. Figure 2 shows the plots of the "root mean square error" (RMSE), computed over $10^{3}$ randomly chosen simulations, for the considered filters. The performance of the FDDN turns out to be better from the point of view of the asymptotic behavior when there is large difference between the disturbance and noise.

\section{Conclusions}

The main contribution of this work is the method of constructing an estimator for the uncertain system with the different state disturbance and measurement noise. The stability of 
the estimator is analyzed using quadratic boundness. Moreover, the estimator can be got by LMI procedures.

\section{Acknowledgments}

This work was supported by National Natural Science Foundation of China under Grant 60974051, Natural Science Foundation of Beijing under Grant 4122071, Fundamental Research Funds for the Central Universities under Grant 12MS143, and the Construction Project from Beijing Municipal Commission of Education. The authors would like to thank the reviewers for their pertinent comments.

\section{References}

[1] J. H. Kim and J. H. Oh, "Robust state estimator of stochastic linear systems with unknown disturbances," IEE Proceedings, vol. 147, no. 2, pp. 224-228, 2000.

[2] M. Fu and C. E. de Souza, "State estimation for linear discrete-time systems using quantized measurements," Automatica, vol. 45, no. 12, pp. 2937-2945, 2009.

[3] B. Boulkroune, M. Darouach, and M. Zasadzinski, "Moving horizon state estimation for linear discrete-time singular systems," IET Control Theory and Applications, vol. 4, no. 3, pp. 339-350, 2010.

[4] S. Pillosu, A. Pisano, and E. Usai1, "Decentralised state estimation for linear systems with unknown inputs: a consensus-based approach," IET Control Theory E Applications, vol. 5, no. 3, pp. 498-506, 2011.

[5] A. Alessandri, M. Baglietto, and G. Battistelli, “Design of state estimators for uncertain linear systems using quadratic boundedness," Automatica, vol. 42, no. 3, pp. 497-502, 2006.

[6] M. L. Brockman and M. Corless, "Quadratic boundedness of nonlinear dynamical systems," in Proceedings of the 34th IEEE Conference on Decision and Control, pp. 504-509, New Orleans, La, USA, December 1995.

[7] M. L. Brockman and M. Corless, "Quadratic boundedness of nominally linear systems," International Journal of Control, vol. 71, no. 6, pp. 1105-1117, 1998.

[8] M. L. Ni and M. J. Er, "Design of linear uncertain systems guaranteeing quadratic boundedness," in Proceedings of the Americal Control Conference, pp. 3832-3836, Chicago, Ill, USA, June 2000.

[9] B. Ding and L. Xie, "Dynamic output feedback robust model predictive control with guaranteed quadratic boundedness," in Proceedings of the 48th IEEE Conference on Decision and Control (CDC/CCC '09), pp. 8034-8039, Shanghai, China, December 2009.

[10] B. Ding, "Quadratic boundedness via dynamic output feedback for constrained nonlinear systems in Takagi-Sugeno's form," Automatica, vol. 45, no. 9, pp. 2093-2098, 2009.

[11] B. Ding, "Dynamic output feedback predictive control for nonlinear systems represented by a takagisugeno model," IEEE Transactions on Fuzzy Systems, vol. 19, no. 5, pp. 831-843, 2011.

[12] B. Ding, Y. Xi, M. T. Cychowski, and T. O’Mahony, "A synthesis approach for output feedback robust constrained model predictive control," Automatica, vol. 44, no. 1, pp. 258-264, 2008.

[13] B. Ding, "New formulation of dynamic output feedback robust model predictive control with guaranteed quadratic boundedness," in Proceedings of the 30th Chinese Control Conference, pp. 33463351, Yantai, China, July 2011.

[14] A. Alessandri, M. Baglietto, and G. Battistelli, “On estimation error bounds for receding-horizon filters using quadratic boundedness," IEEE Transactions on Automatic Control, vol. 49, no. 8, pp. 13501355, 2004.

[15] M. Chilali and P. Gahinet, " $\mathrm{H}_{\infty}$ design with pole placement constraints: an LMI approach," IEEE Transactions on Automatic Control, vol. 41, no. 3, pp. 358-367, 1996. 


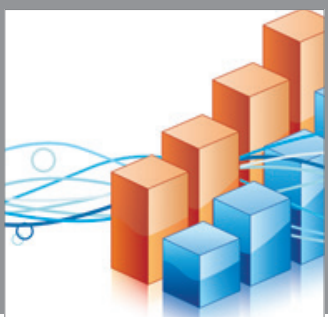

Advances in

Operations Research

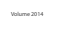

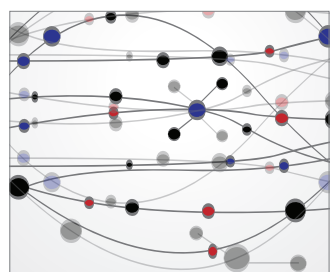

\section{The Scientific} World Journal
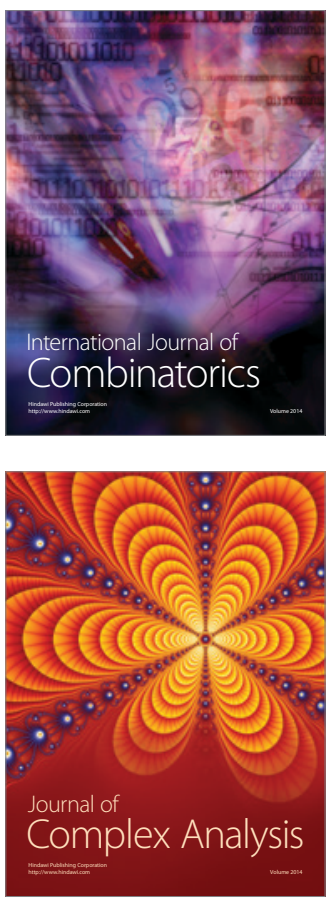

International Journal of

Mathematics and

Mathematical

Sciences
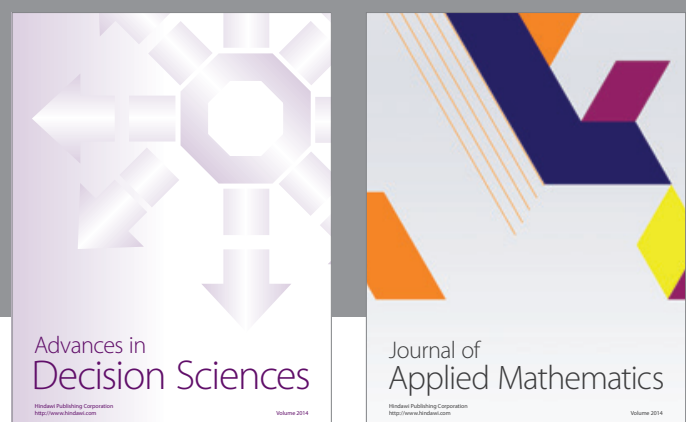

Journal of

Applied Mathematics
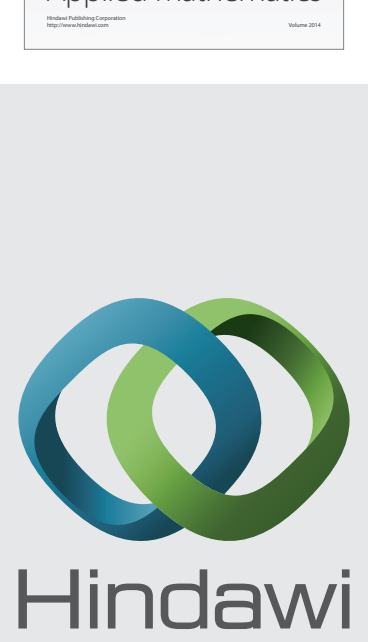

Submit your manuscripts at http://www.hindawi.com
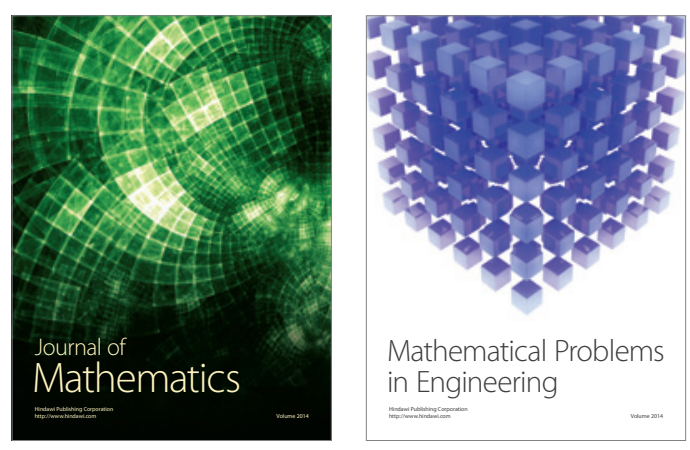

Mathematical Problems in Engineering
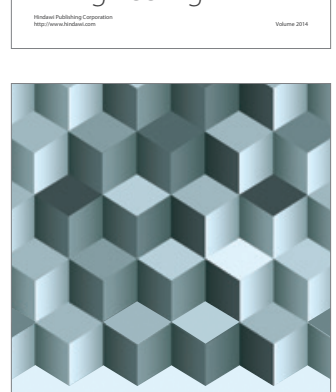

Journal of

Function Spaces
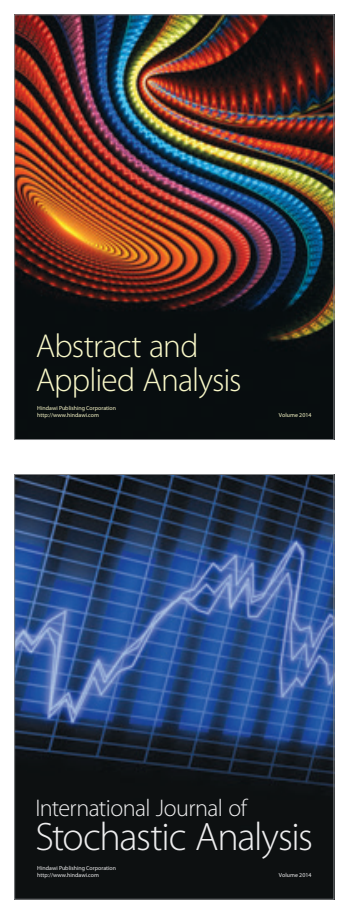

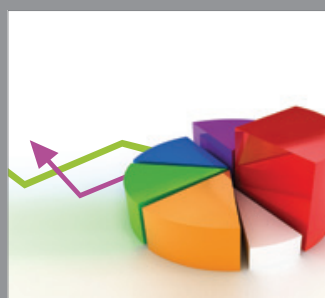

ournal of

Probability and Statistics

Promensencen
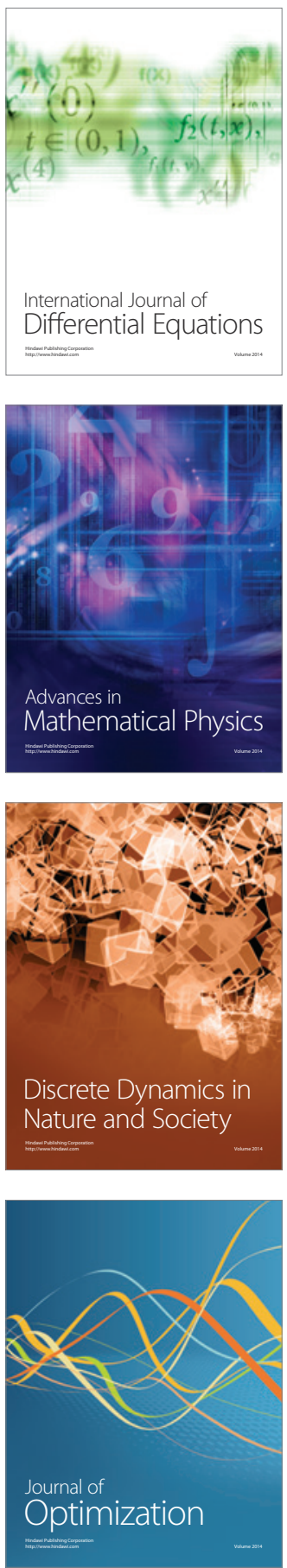